\title{
Study on the Value of Visualized Immediate Feedback of Interior Bare-handed Expression Technique in the Information Era
}

\author{
Zhou Zewen, Lu Ying \\ Guangzhou College of Technology and Business, Guangzhou, Guangdong, 510850, China
}

Keywords: Interior Design; FMCG; Bare-handed Expression; Immediate Feedback; Visualized

\begin{abstract}
In the information era, services in the home decoration industry become "fast-moving consumer goods (FMCGs)" due to streamlined design with high design software operation skills: modularized furniture combination and allocation, set materials and processes and increasingly identical design ideas. After a round of market competition, the market pattern is reshuffled where market demand for talents is no longer restricted to technology; instead, "creative thinking" and "differentiated services" are highlighted among enterprises. Design is concerned with problem solving in immediate reception and feedback in nature, wherein, field survey and communication are crucial design services. Designers can give visualized immediate information feedback concerning problems encountered, reduce expression and communication costs and establish an effective exchange with customers and working teams by the bare-handed expression ability.
\end{abstract}

\section{Introduction}

The combination of on- and off-line business models in the home decoration design industry gives rise to "popularization" and "FMCG" trends in the home space design industry. On one hand, main market consumers acquire design image, text and design information on the Internet, accumulate a certain amount of professional knowledge, prefer a simple design, and highlight environmental protection and construction quality, whose demand presents a reasonable tendency due to "popularization" of design knowledge; on the other hand, "FMCG" home decoration shops have sprung up one after another under the impact of real estate economy, leading to a market saturation. As a result, many shops have failed and closed in the competition. The explosion of "FMCG" home decoration firms indicates repetition and focus on quantity rather than quality in design which does not match such mounting needs of main consumers as rationalization, craftsmanship and personalization. There is no effective emotional communication between main consumers and home decoration firms!

\section{Cost of the Working Model of Interior Designers}

An interior designer uses the bare-handed expression technique to prepare the Round 1 proposal, upon approval of which, the designer details the design proposal by design software, wherein, bare-handed expression requires a hand-eye coordination (what you see is what you get) and minimizes the cost of financial and material resources; the designer uses design software to detail design, build a model and deliberate the proposal, which is time-consuming and risky for building a real or computer model under many uncertain preliminary factors in the project; in deliberation of the proposal, the designer uses 3D software drawing effects to reflect a real environment, which, whereas, does not facilitate problems that stand out to be expressed in time or establish an effective communication herein; in software rendering production, the design team has a high communication cost and overtime becomes a working normal.

\section{Feedback of Interior Bare-handed Expression}

Used to be merely a concept in physics, feedback refers to an effect of sending a certain amount of energy in the output circuit of amplifier back to the input circuit to reinforce or weaken input 
signals. This concept is taken for reference in psychology to describe a learner's understanding about his/her own learning results which intensifies and facilitates the learner to study harder to enhance learning efficiency. This psychological phenomenon is termed "feedback".

As a matter of fact, the overtime working normal of interior designers objectively indicates a high time cost, wherein, design proposals are ineffectively and repeatedly revised at an inefficient stage. As far as designers adopting an "FMCG" working model are concerned, they spend less time in making face-to-face communication with customers and conducting a survey on customers' habits in life, hence they mostly give dimensions in a conventional pattern and slightly adjust spatial structure in proposals and seldom show elaborate and customer-friendly design, the key cause of which is lack of effective information feedback.

In contrast, excellent designers show their high working efficiency in the following aspects: they fulfill both functional and mental needs in design; they record customers' needs at length by image-text visualized interior bare-handed expression drawings while describing preliminary design proposals concerning the former status of space and directly receiving information feedback from customers in communication with customers to further accurately grasp and adjust actual needs of customers.

\section{Immediate Feedback of Bare-handed Expression of Interior Designers}

A psychologist Hurlock did a psychological experiment on "feedback" of four teams which finished work inspired by different incentives. Its findings are as follows: persons have better learning effects under information feedback than ones without feedback in life and a timely evaluation and learning and activity results can strengthen the learning and activity motivations to boost working; immediate feedback has greater effects than long-term feedback. This effect reminds that effective feedback mechanism is an essential condition for hitting the activity target.

\subsection{Immediate feedback from customers}

In practical work, designers base their initial design work on a two-way survey on target persons and circumstances, make a face-to-face communication, take photos of routine life, and measure such environmental coefficients as air and water quality by electronic equipment to get a defined design basis. First of all, data feedback of objective needs are given in real time, which help designers build a framework in mind and initially defines the proportion of practical functions, aesthetic needs, technical support and economic cost. Secondly, an immediate communication with customers helps designers quickly sort out differences between designers and customers in subjective perception. Everyone has his/her own understanding about specific terms and a professional oral description seems too abstract. In order to ensure an effective communication, interior bare-handed expression drawings are needed to directly eliminate understanding differences and give clear answers to customers' questions with a purpose. Last but not least, customers need evaluate activity results in time, which can reinforce the activity motivation, prevent problems from accumulating and boost working.

\subsection{Self-immediate-feedback of designers}

The coordination in a design team is shown in awareness of design objectives and working abilities of members in the team and transmission and reception of information comes first in connecting and detailing tasks of all the working squads in the team. To boost a proposal in design work is required of ongoing discussion and study for it is a process which takes a certain amount of time to produce results. The results referred to include design understanding ability, aesthetic appreciation ability, problem-solving communication mode and teamwork mechanism, which require the design team to establish immediate feedback on a constant basis to urge itself. In detailing design drawings in preliminary proposal and preparation of working drawings and rendering for instance, each staged design director need consider which ways to be taken to describe the design philosophy, details of implementation of proposal, and how to accurately elaborate requirements for such data as dimensions, structure, materials, and colors in design. In that case, 
interior bare-handed expression technique helps create a detailed and specific communication by quick transmission of such data information as image, text and figure. Similarly, bare-handed expression also plays a crucial role in the self-feedback stage where designers optimize and boost design details by establishing a barrier-free communication and enhancing efficiency in deliberation of proposal and allocation of working hours in a tight schedule.

\section{Visualization of Language Information by Interior Bare-handed Expression}

Assuming that an effective information feedback activity is an essential condition for fulfilling objectives, interior bare-handed expression as an instrument for implementation of demand for immediate feedback of the design activity must have its value of existence.

It is an ability to accurately output personal thinking and language information in a simple way at work and "picture" is something in common that people share in terms of self-cognition in normal life. In front of a target of communication, one can give a clarity to functional properties by roughly and repeatedly sketching lines instead of drawing details in illustration of interior bare-handed expression. The conventional interior bare-handed expression at the learning stage is aimed at making true-life drawings in presentation of proposal. In the wake of development of information technology, increasingly modularized design rendering production software can quickly and precisely reflect this function. Later at the "FMCG" design service stage, the interior bare-handed expression technique is underestimated by designers and even has no room for showing its practical value. Out of the scope of "FMCG" design and emotional detail design, the bare-handed expression technique exists in a form that breaks away from the representation of perfect rendering, gives a description where pictures predominate with such aids as figure and text, reflect the feasibility of design details, and cover scale of functional space, categories of materials in combination, color and emotional needs, details of construction nodes and other data communication and discussion results, which is a full range of description records for the design process. Therefore, drawing software reforms in the Internet era cannot deny the value of existence of interior bare-handed expression technique; instead, the above two are supposed to work together to boost industrial development.

\section{Conclusions}

In the Internet-based information era, the interior bare-handed expression technique will exist in a variety of forms and facilitate interior designers to do the design work by replacing the conventional paper media by tablet PC and combining with creative bare-handed drawing software. Bare-handed design drawings can be called whenever possible from computer records and carried in an easier and more eco-friendly way and information therein can be more easily modified and transmitted compared with the conventional paper media. Nonetheless, the fundamental carrier of the bare-handed expression technique is the human thought albeit the media change and service aims to establish an effective inter-individual, individual-collective and individual-self communication and connection to a certain extent of cognition to meet design needs. Therefore, the key value of interior bare-handed expression that is thought highly of in the design industry is the visualized immediate feedback!

\section{References}

[1] Zhuang Xijian, 60 Universal Psychological Principles [M], Beijing: Enterprise Management Publishing House. January 2010

[2] Gao Lihong, Reinforcing Learning Effects by Feedback [J]. Campus Life \& Mental Health, 2007, (6).

[3] Li Yonggang, A Discussion on How to Motivate and Train Students' Learning Interest [J], Journal of Pingyuan University, 2001, (1): 91-92 